CARDIOVASCULAR MEDICINE

\title{
Use of oral glucocorticoids and risk of cardiovascular and cerebrovascular disease in a population based case-control study
}

\author{
P C Souverein, A Berard, T P Van Staa, C Cooper, A C G Egberts, H G M Leufkens, B R Walker
}

Heart 2004;90:859-865. doi: 10.1136/hrt.2003.020180

See end of article for authors' affiliations .....................

Correspondence to: Dr P C Souverein Department of Pharmacoepidemiology and Pharmacotherapy, Utrecht Institute for Pharmaceutical Sciences, PO Box 80082, 3508 TB Utrecht, Netherlands; P.C.Souverein@ pharm.uu.nl

Accepted 28 November 2003
Objective: To assess whether use of oral glucocorticoids is associated with cardiovascular and cerebrovascular morbidity.

Design and setting: Nested case-control study within a cohort of patients ( $\geqslant 50$ years old) with at least one prescription for oral or non-systemic glucocorticoids. Data were from the general practice research database.

Patients: 50656 patients were identified with a first record for ischaemic heart disease (International classification of diseases, ninth revision (ICD-9) codes 410,411,413, and 414), ischaemic stroke or transient ischaemic attack (ICD-9 codes 430-436), or heart failure (ICD-9 code 428) between 1988 and 1998. One control was matched to each case by sex, age, general practice, underlying disease, and calendar time.

Main outcome measure: Odds ratio (OR) of cardiovascular or cerebrovascular events in patients using oral glucocorticoids compared with non-users.

Results: There was a significant association between ever use of oral glucocorticoids and any cardiovascular or cerebrovascular outcome (adjusted OR 1.25, 95\% confidence interval (Cl) 1.21 to 1.29). The association was stronger for current use of oral glucocorticoids than for recent or past use. Among current users, the highest ORs were observed in the group with the highest average daily dose, although the dose-response relation was not continuous. Current use was associated with an increased risk of heart failure (adjusted OR 2.66, 95\% Cl 2.46 to 2.87), which was consistent between patients with rheumatoid arthritis, patients with chronic obstructive pulmonary disease, and patients without either of the two conditions. Also, current use was associated with a smaller increased risk of ischaemic heart disease (OR $1.20,95 \% \mathrm{Cl} 1.11$ to 1.29 ).

Conclusions: Oral glucocorticoid use was identified as a risk factor for heart failure. However, the evidence remains observational and only a randomised controlled trial of glucocorticoid treatment versus other disease modifying agents is likely to distinguish the importance of the underlying disease activity from its treatment in predicting cardiovascular outcomes.
G lucocorticoids are widely prescribed drugs in modern medicine. Data from the UK suggest that nearly $1 \%$ of the total adult population use oral glucocorticoids. ${ }^{1}$ Use of oral glucocorticoids has been associated with the occurrence of side effects, such as increased risk of fracture, ${ }^{2}$ but there is also concern with respect to metabolic and cardiovascular morbidity and mortality. ${ }^{3}$ In addition, glucocorticoid use has been shown to be associated with fluid retention, which is induced by an enhanced sodium reabsorption and expansion of the extracellular fluid volume. ${ }^{5}$ Also, patients with Cushing's syndrome have an increased risk of obesity, hypertension, hyperglycaemia, and premature death caused by high plasma concentrations of the endogenous glucocorticoid cortisol. ${ }^{6}$ There is also evidence that, even among men with cortisol secretion within the "normal" range, hypercortisolism is associated with risk factors for cardiovascular and cerebrovascular outcomes, including hypertension, hyperglycaemia, and dyslipidaemia. ${ }^{7-14}$ However, not all patients with these risk factors develop cardiovascular and cerebrovascular diseases. Indeed, atherosclerosis is increasingly recognised as an inflammatory condition of the blood vessel wall. ${ }^{15}$ Glucocorticoids have anti-inflammatory and antiproliferative actions in vessels, ${ }^{16} 17$ which may counteract their adverse effects on systemic cardiovascular and cerebrovascular risk factors.
Against this background, it has yet to be established whether use of exogenous glucocorticoids is associated with an increased risk of cardiovascular and cerebrovascular morbidity. Very few randomised controlled trials have ever been performed with oral glucocorticoid treatment and none has been powered to detect differences in cardiovascular events. ${ }^{18}$ Some of the disorders for which glucocorticoids are commonly prescribed are themselves associated with cardiovascular disease. Patients with rheumatoid arthritis (RA) or chronic obstructive pulmonary disease (COPD) have an increased risk of cardiovascular mortality due to the systemic effect of the disease, regardless of the medications they used. ${ }^{19-23}$ However, it is unclear whether use of glucocorticoids contributes to these associations.

Hence, the objective of this study was to quantify the association between use of oral glucocorticoids and cardiovascular and cerebrovascular morbidity in a nested casecontrol study. Data were obtained from the general practice research database (GPRD).

Abbreviations: $\mathrm{BMI}$, body mass index; $\mathrm{Cl}$, confidence interval; $\mathrm{COPD}$ chronic obstructive pulmonary disease; GPRD, general practice research database; ICD-9, International classification of diseases, ninth revision; NSAIDs, non-steroidal anti-inflammatory drugs; OR, odds ratio; RA, rheumatoid arthritis 


\section{METHODS \\ Setting}

General practitioners have a key role in the health care system in the UK, as they are responsible for primary health care and specialist referrals. Patients are semipermanently affiliated to a practice, which centralises the medical information not only from the general practitioners themselves but also from specialist referrals and hospitalisations. The current study drew data from 683 practices in various geographical areas in the UK registered on the GPRD.

The GPRD covers a population of about 3.5 million men and women. The data recorded on the GPRD include demographic information, prescription data, clinical events, preventive care provided, specialist referrals, hospital admissions, and their major outcomes. ${ }^{24}$ Clinical data are stored and retrieved by means of the Oxford medical information system and read codes for diseases that are cross referenced to the International classification of diseases, ninth revision (ICD-9). Each entry on the GPRD is internally validated by cross checking within the practice and by comparisons with external statistics. ${ }^{24}$ Only data from practices that pass this quality control are compiled and are part of the GPRD. Several independent validation studies have confirmed a high level of completeness and validity of the GPRD, which is owned by the Department of Health in the UK. ${ }^{25}$

\section{Study population}

From the GPRD, we identified all permanently registered patients aged 50 years and older with at least one prescription for oral glucocorticoids during the time from the enrolment date of their practice in the GPRD (data collection started in 1988) to the end of the study period (31 December 1997), as well as patients who received at least one prescription for non-systemic glucocorticoids (topical, aural, ophthalmic, or nasal) but not for systemic glucocorticoids. Use of inhaled steroids was also allowed for patients using oral glucocorticoids. The date of the first corticosteroid prescription marked the start of follow up. The follow up of the patient stopped when the patient died or was lost to follow up, at the occurrence of an event (see list of events below), or at the end of the study period (December 1997), whichever occurred first.

Documented prescription is a means of ensuring active registration of participants in GPRD. To avoid biasing the results by inclusion of patients for whom data collection was incomplete, all participants were required to receive at least one prescription for glucocorticoids during the study period.

\section{Definition of cases and controls}

Within the cohort of glucocorticoid users, a nested casecontrol study was conducted. Cases were defined as patients with a first record of ischaemic heart disease (ICD-9 code $410,411,413,414$ ), heart failure (ICD-9 code 428), or ischaemic stroke or transient ischaemic attack (ICD-9 code 430-436) during follow up. The date of the first event during follow up was the index date for the cases. For each case, one control was matched on sex, year of birth, general practice, presence of underlying disease, and calendar time. When no eligible control was found, the matching criterion with respect to year of birth was relaxed (to \pm 3 years). When it was still impossible to find a suitable control, a control from another practice was chosen. Controls were eligible for inclusion if they had no record of ischaemic heart disease, cerebrovascular events, or heart failure at any time.

Because of the possible association between indications for glucocorticoids and cardiovascular disease, cases and controls were matched for the presence or absence of these underlying diseases. Patients in the case and control groups were classified as having RA (ICD-9 code 714.0) if they had RA diagnosed before the index date, and were classified as having COPD or allied conditions (ICD-9 codes 490-496) if they had COPD diagnosed before the index date. The date of the first recording of RA or COPD diagnosis was used to estimate the duration of disease. Patients without recorded diagnoses of RA or COPD were included only if they were not using prescription drugs indicating the presence of either disease. Patients who had both diagnoses of RA and COPD contributed to both the RA group and the COPD group.

\section{Exposure assessment}

For each patient, we identified all prescriptions for oral (systemic) glucocorticoids (cortisone, hydrocortisone, prednisone, prednisolone, triamcinolone, methylprednisone, dexamethasone, and betamethasone) between entry into the cohort and the index date. Several indices of oral glucocorticoid exposure were calculated. Exposure was categorised dichotomously according to whether oral glucocorticoids had been prescribed before the index date. Exposure was also classified according to timing of use in relation to the index date: "current use" was defined as any prescription of oral glucocorticoids in the three month period before the index date; "recent use" was defined as having no prescription in the three month period before the index date and a prescription one year before the index date; and "past use" was defined as no prescriptions during one year before the index date. Oral glucocorticoid doses were calculated as prednisolone equivalent doses, where $5 \mathrm{mg}$ prednisolone was equivalent to $25 \mathrm{mg}$ cortisone, $20 \mathrm{mg}$ hydrocortisone, $5 \mathrm{mg}$ prednisone, $4 \mathrm{mg}$ triamcinolone, $4 \mathrm{mg}$ methylprednisone, $750 \mu \mathrm{g}$ dexamethasone, or $750 \mu \mathrm{g}$ betamethasone. The average daily dose of oral glucocorticoids for current, recent, and past users was calculated from data on the prescribed daily dose that was available from the general practitioner records. The average daily dose was classified as missing if less than $50 \%$ of the prescribed daily doses for the patient were not recorded on the database. The cumulative dose was obtained by summing all doses and was classified as missing when one or more doses were not registered on the database.

\section{Assessment of potential confounders}

Potential confounders in this study were medical conditions and drugs that have been associated with an increased or decreased risk of cardiovascular and cerebrovascular disease. To address confounding by co-prescription of other drugs, we assessed use of antihypertensive drugs (diuretics, $\beta$ blockers, angiotensin converting enzyme inhibitors, calcium channel blockers, or other), use of lipid lowering drugs (fibrates or statins), nitrates, cardiac glycosides, antiplatelet drugs, oral anticoagulants, and antiarrhythmics, as well as use of insulin, oral hypoglycaemic drugs, and hormone replacement therapy in the year before the index date. In addition, we assessed the use of other drugs indicated for the treatment of RA (non-steroidal anti-inflammatory drugs (NSAIDs) and disease modifying anti-rheumatic drugs) and lung disease (bronchodilators, cromoglycates) in the year before the index date.

Confounding by disease indication was addressed by examining consistency of effects in cases and controls matched for RA, COPD, and other indications. The number of physician visits (general practitioner or specialist) and number of hospital admissions in the year before the start of glucocorticoid use were also considered as markers for patients' health status. Further, we evaluated the effect of smoking and body mass index (BMI).

\section{Data analysis}

The strength of the association between use of oral glucocorticoids and the occurrence of outcome events 
(ischaemic heart disease, stroke/transient ischaemic event, and heart failure) was estimated by conditional logistic regression analysis and expressed as crude and adjusted odds ratios (OR) and 95\% confidence intervals (CI). Covariates were included in the regression model if they were either independently significantly associated with the outcome or induced a $10 \%$ or greater change in the crude matched OR for oral corticosteroid use. ${ }^{26}$ In addition, ischaemic heart disease, heart failure, and cerebrovascular events were analysed separately. Patients with RA, patients with COPD, and patients without either of these conditions were analysed as subgroups. As entering information on BMI and smoking is optional for the general practitioner, data were analysed on patients with complete information only, as well as on all patients with the use of an indicator for missing data. As the results of both analyses were not different, BMI and smoking of all patients were adjusted for in the final analyses.

\section{RESULTS}

The study population comprised 50656 patients and an equal number of matched controls. Table 1 lists the characteristics of the study population. The majority were women $(56.7 \%)$ and about $85 \%$ were aged between $60-90$ years. There were 1515 case-control pairs matched for diagnosis of RA (median duration of disease among cases and controls 7.2 years and 6.8 years, respectively), 16440 case-control pairs matched for diagnosis of asthma or COPD (median duration 3.7 years for both cases and controls), and 31240 case-control pairs without a diagnosis of RA or COPD. High BMI and smoking were significantly more frequent among the cases than among controls, as was the prevalence of prescription drug use in the year before the index date. The most frequently used drugs were NSAIDs, diuretics, bronchodilators, and $\beta$ blockers. Further, the numbers of hospital admissions and general practitioner visits in the year before the index date were higher among cases than among controls.

Table 2 shows the association between the use of oral glucocorticoids and the risk of any cardiovascular or cerebrovascular outcome. The prevalence of using oral glucocorticoids before the index date was higher among the cases $(30.3 \%)$ than among controls $(26.4 \%)$, yielding a crude OR of 1.31 (95\% CI 1.27 to 1.35). This relation persisted after adjusting for use of NSAIDs, hormone replacement therapy, antihypertensive drugs, nitrates, oral anticoagulants, antiplatelet drugs, antidiabetic drugs, bronchodilators, cromoglycates, inhaled steroids, disease modifying antirheumatic drugs, smoking, and BMI (adjusted OR 1.25, 95\% CI 1.21 to 1.29). The association was stronger for current use of oral

Table 1 Characteristics of cases and matched controls

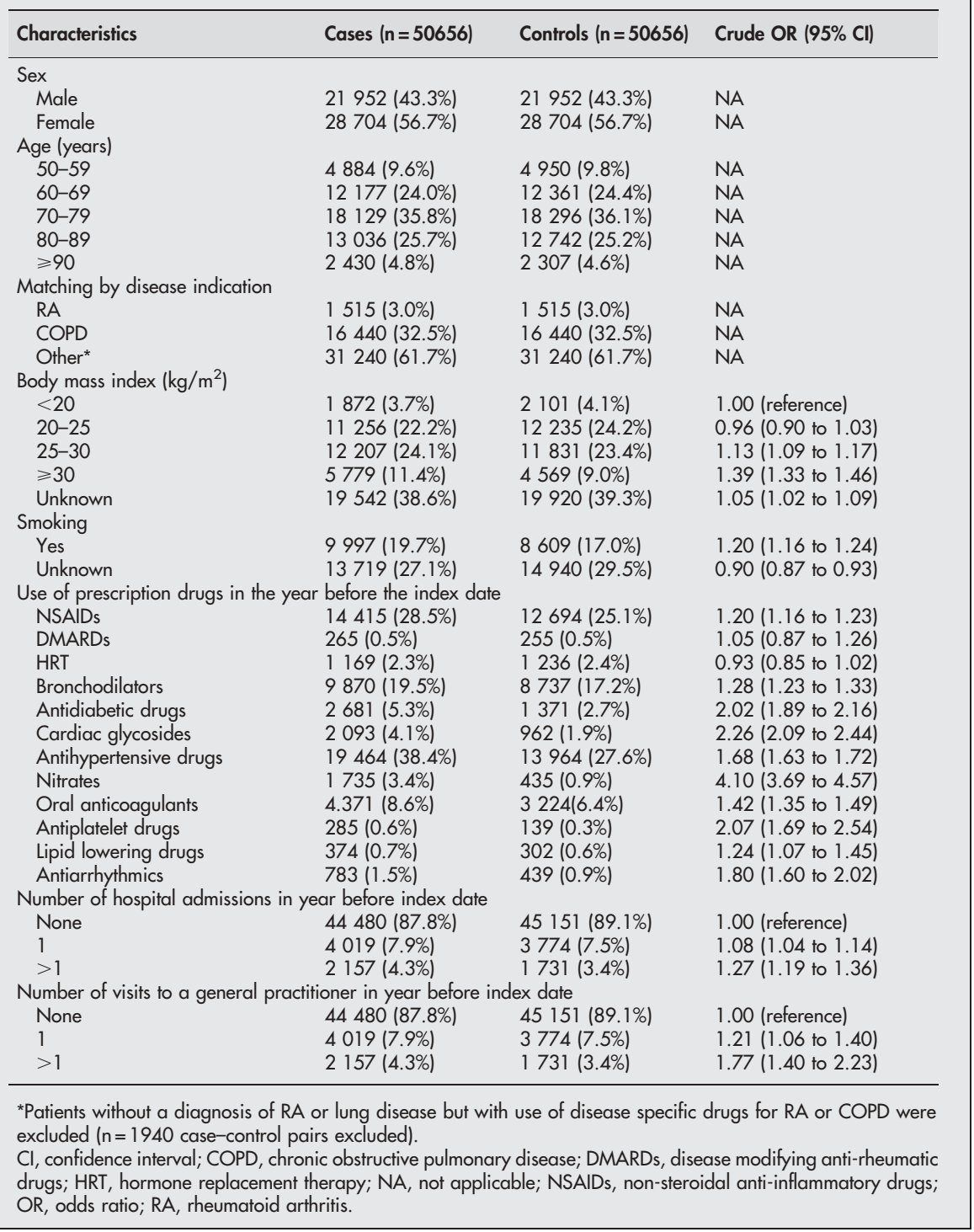


Table 2 Exposure to oral glucocorticoids among cases $(n=50656)$ and controls $(n=50656)$ and risk of any cardiovascular or cerebrovascular event

\begin{tabular}{|c|c|c|c|c|}
\hline Exposure & Cases $(n=50656)$ & $\begin{array}{l}\text { Controls } \\
(n=50 \text { 656) }\end{array}$ & Crude OR $(95 \% \mathrm{Cl})$ & Adjusted OR $(95 \% \mathrm{Cl})^{*}$ \\
\hline \multicolumn{5}{|c|}{ Ever used oral glucocorticoids } \\
\hline Yes & 15355 (30.3\%) & $13395(26.4 \%)$ & 1.31 (1.27 to 1.35$)$ & 1.25 (1.21 to 1.29$)$ \\
\hline \multicolumn{5}{|c|}{ Timing of oral glucocorticoid use } \\
\hline Current use & $8167(16.1 \%)$ & $6015(11.9 \%)$ & $1.56(1.50$ to 1.62$)$ & $1.48(1.41$ to 1.54$)$ \\
\hline Recent use & $3491(6.9 \%)$ & $3681(7.3 \%)$ & $1.08(1.02$ to 1.14$)$ & $1.03(0.98$ to 1.09$)$ \\
\hline Past use & $3697(7.3 \%)$ & 3699 (7.3\%) & $1.12(1.06$ to 1.18$)$ & $1.08(1.02$ to 1.14$)$ \\
\hline \multicolumn{5}{|c|}{ Current users of oral glucocorticoids (average daily dose in $\mathrm{mg}$ prednisolone equivalents) } \\
\hline$<7.5 \mathrm{mg} /$ day & $2211(4.5 \%)$ & $1710(3.4 \%)$ & $1.46(1.36$ to 1.57$)$ & $1.44(1.34$ to 1.55$)$ \\
\hline $7.5-20 \mathrm{mg} / \mathrm{da} y$ & $2015(4.1 \%)$ & $1627(3.3 \%)$ & 1.37 (1.28 to 1.48$)$ & $1.30(1.21$ to 1.40$)$ \\
\hline$\geqslant 20 \mathrm{mg}$ & $2593(5.3 \%)$ & $1735(3.5 \%)$ & $1.73(1.62$ to 1.86$)$ & $1.63(1.52$ to 1.75$)$ \\
\hline \multicolumn{5}{|c|}{ Quartiles of cumulative dose (in mg prednisolone equivalents) } \\
\hline$<500 \mathrm{mg}$ & $1925(4.4 \%)$ & $1362(3.2 \%)$ & $1.58(1.45$ to 1.73$)$ & 1.61 (1.38 to 1.88$)$ \\
\hline $500-1810 \mathrm{mg}$ & $2047(4.7 \%)$ & $1605(3.7 \%)$ & $1.39(1.28$ to 1.51$)$ & 1.34 (1.16 to 1.54$)$ \\
\hline $1810-5418 \mathrm{mg}$ & $2006(4.6 \%)$ & 1649 (3.8\%) & $1.36(1.26$ to 1.47$)$ & 1.19 (1.03 to 1.37$)$ \\
\hline$>5418 \mathrm{mg}$ & $2009(4.6 \%)$ & $1277(3.0 \%)$ & 1.71 (1.57 to 1.86$)$ & $1.56(1.35$ to 1.80$)$ \\
\hline
\end{tabular}

glucocorticoids than for recent or past use. Among current users, the highest ORs were observed in the group with the highest average daily dose, although the dose-response relation was not continuous. There was no clear association with cumulative dose.

Analysis according to the type of outcome event indicated that current use of oral glucocorticoids was associated with an increased risk for heart failure (adjusted OR 2.66, 95\% CI 2.46 to 2.87) (table 3). Further, current use of oral glucocorticoids was associated with an increased risk for ischaemic heart disease events (adjusted OR 1.20, 95\% CI 1.11 to 1.29 ) but was associated with a decreased risk for cerebrovascular disease (adjusted OR 0.91, 95\% CI 0.84 to 0.99). To explore further the association between heart failure and use of glucocorticoids, we stratified according to daily dose.

There was a dose-response relation between daily dose and risk of heart failure among current users of oral glucocorticoids (adjusted OR 1.95, 95\% CI 1.72 to 2.21 for low dose ( $<7.5 \mathrm{mg}$ prednisolone equivalent daily dose); adjusted OR 2.27, $95 \%$ CI 2.00 to 2.59 for medium dose $(7.5-20 \mathrm{mg}$ prednisolone equivalent daily dose); and adjusted OR 3.69, 95\% CI 3.26 to 4.18 for high dose (>20 mg prednisolone equivalent daily dose). Stratification according to history of cardiovascular drug use showed that the stratum specific ORs were similar for patients with and those without a history of cardiovascular drug use and use of antidiabetic drugs (data not shown).

To explore the possibility that certain covariates differed between users and non-users, a logistic regression model was fitted with use of glucocorticoids as dependent variable and patient characteristics, health care utilisation, and use of prescription drugs as independent variables. We found that patients using antidiabetic drugs were less likely to use oral glucocorticoids (OR 0.61, 95\% CI 0.56 to 0.67 ).

Current use of oral glucocorticoids was associated with heart failure in all groups: the RA matched group, the COPD matched group, and the non-RA/non-COPD matched group (table 4). However, the relation was strongest in the COPD group. For ischaemic heart disease, current use of oral glucocorticoids was similarly increased in the RA matched group and the COPD matched group. In the group of patients

Table 3 Use of oral glucocorticoids and cardiovascular or cerebrovascular events, stratified according to type of outcome event

\begin{tabular}{|c|c|c|c|c|}
\hline Outcome event & Cases $(n=50656)$ & Controls ( $n=50656$ ) & Crude OR $(95 \% \mathrm{Cl})$ & Adjusted OR $(95 \% \mathrm{Cl})^{*}$ \\
\hline \multicolumn{5}{|c|}{ Ischaemic heart disease } \\
\hline \multicolumn{5}{|c|}{ Ever used oral glucocorticoids } \\
\hline Yes & $5298(25.6 \%)$ & $4956(24.0 \%)$ & $1.13(1.08$ to 1.20$)$ & $1.09(1.03$ to 1.15$)$ \\
\hline \multicolumn{5}{|c|}{ Timing of oral glucocorticoid use } \\
\hline Current use & $2507(12.1 \%)$ & 2115 (20.2\%) & 1.26 (1.18 to 1.35$)$ & $1.20(1.11$ to 1.29$)$ \\
\hline Recent use & $1310(6.3 \%)$ & $1422(6.9 \%)$ & 0.98 (0.90 to 1.06 ) & 0.93 (0.85 to 1.02 ) \\
\hline Past use & $1481(7.2 \%)$ & $1419(6.9 \%)$ & $1.10(1.01$ to 1.19$)$ & $1.07(0.98$ to 1.17$)$ \\
\hline \multicolumn{5}{|l|}{ Heart failure } \\
\hline \multicolumn{5}{|c|}{ Ever used oral glucocorticoids } \\
\hline Yes & 6401 (44.4\%) & 4763 (33.1\%) & 2.00 (1.88 to 2.12 ) & 1.91 (1.79 to 2.03 ) \\
\hline \multicolumn{5}{|c|}{ Timing of oral glucocorticoid use } \\
\hline Current use & $4020(27.9 \%)$ & 2209 (15.3\%) & 2.77 (2.57 to 2.97 ) & 2.66 (2.46 to 2.87$)$ \\
\hline Recent use & $1299(9.0 \%)$ & 1315 (9.1\%) & 1.48 (1.35 to 1.62 ) & $1.40(1.27$ to 1.55$)$ \\
\hline Past use & $1082(7.5 \%)$ & $1239(8.6 \%)$ & $1.23(1.12$ to 1.36$)$ & $1.19(1.08$ to 1.32$)$ \\
\hline \multirow{2}{*}{\multicolumn{5}{|c|}{$\begin{array}{l}\text { Stroke/transient ischaemic attack } \\
\text { Ever used oral alucocorticoids }\end{array}$}} \\
\hline & & & & \\
\hline Yes & $3656(23.4 \%)$ & $3676(23.6 \%)$ & $0.99(0.93$ to 1.05$)$ & 0.95 (0.89 to 1.01$)$ \\
\hline \multicolumn{5}{|c|}{ Timing of oral glucocorticoid use } \\
\hline Current use & $1640(10.5 \%)$ & 1691 (10.8\%) & 0.96 (0.89 to 1.04 ) & 0.91 (0.84 to 0.99$)$ \\
\hline Recent use & $882(5.7 \%)$ & $944(6.1 \%)$ & 0.93 (0.84 to 1.03 ) & $0.89(0.80$ to 0.99$)$ \\
\hline Past use & $1134(7.3 \%)$ & $1041(6.7 \%)$ & 1.09 (0.99 to 1.20$)$ & $1.06(0.96$ to 1.17$)$ \\
\hline
\end{tabular}


Table 4 Use of oral glucocorticoids and cardiovascular or cerebrovascular events stratified by disease indication: rheumatoid arthritis (RA, $n=1515)$, chronic obstructive pulmonary disease (COPD, $n=16440)$, other $(n=31240)$

\begin{tabular}{|c|c|c|c|}
\hline & \multicolumn{3}{|l|}{ Adjusted OR $(95 \% \mathrm{Cl})$} \\
\hline & RA cohort* & COPD cohortt & Other cohortt \\
\hline \multicolumn{4}{|l|}{ All outcomes } \\
\hline \multicolumn{4}{|c|}{ Timing of oral glucocorticoid use } \\
\hline Current use & 1.37 (1.16 to 1.62$)$ & $1.68(1.57$ to 1.80$)$ & $1.13(0.99$ to 1.29$)$ \\
\hline Recent use & $1.28(0.98$ to 1.68$)$ & $1.06(0.98$ to 1.15$)$ & $1.12(0.92$ to 1.35$)$ \\
\hline Past use & 1.17 (0.87 to 1.58$)$ & $1.08(1.00$ to 1.16$)$ & $1.13(0.95$ to 1.33$)$ \\
\hline \multicolumn{4}{|c|}{ Ischaemic heart disease events } \\
\hline \multicolumn{4}{|c|}{ Timing of oral glucocorticoid use } \\
\hline Current use & $1.36(1.02$ to 1.81$)$ & 1.28 (1.06 to 1.55$)$ & 1.09 (0.96 to 1.22$)$ \\
\hline Recent use & 1.09 (0.67 to 1.77 ) & 0.94 (0.77 to 1.14 ) & $0.97(0.81$ to 1.17$)$ \\
\hline Past use & $1.34(0.82$ to 2.19$)$ & 1.05 (0.87 to 1.26$)$ & $1.12(0.95$ to 1.32$)$ \\
\hline \multicolumn{4}{|l|}{ Heart failure } \\
\hline \multicolumn{4}{|c|}{ Timing of oral glucocorticoid use } \\
\hline Current use & 1.55 (1.16 to 2.07$)$ & 3.03 (2.69 to 3.40$)$ & $1.89(1.65$ to 2.16$)$ \\
\hline Recent use & 1.52 (0.98 to 2.33$)$ & 1.39 (1.22 to 1.58$)$ & $1.38(1.10$ to 1.74$)$ \\
\hline Past use & $1.12(0.69$ to 1.84$)$ & $1.14(1.00$ to 1.30$)$ & $1.42(1.14$ to 1.77$)$ \\
\hline \multicolumn{4}{|c|}{ Stroke/transient ischaemic attack } \\
\hline \multicolumn{4}{|c|}{ Timing of oral glucocorticoid use } \\
\hline Current use & 1.23 (0.92 to 1.64$)$ & $0.81(0.71$ to 0.93$)$ & $1.03(0.92$ to 1.16$)$ \\
\hline Recent use & $1.23(0.73$ to 2.08$)$ & 0.88 (0.76 to 1.02 ) & $0.93(0.77$ to 1.13$)$ \\
\hline Past use & 1.07 (0.60 to 1.91$)$ & 1.00 (0.87 to 1.16$)$ & $1.29(1.09$ to 1.53$)$ \\
\hline \multicolumn{4}{|c|}{$\begin{array}{l}\text { *Adjusted for use antidiabetic drugs, cardiovascular drugs, NSAIDs, DMARDs, and history or treatment of lung } \\
\text { diseases; tadjusted for use of antidiabetic drugs, cardiovascular drugs, NSAIDs, number of general practitioner } \\
\text { visits, BMI, and smoking. }\end{array}$} \\
\hline
\end{tabular}

without RA and lung disease, use of oral glucocorticoids was not significantly associated with ischaemic heart disease (adjusted OR $1.09,95 \%$ CI 0.96 to 1.22 ). There was no association between oral glucocorticoid use and cerebrovascular events in any of the case-control patient subgroups.

\section{DISCUSSION}

This large nested case-control study addressed specifically the risk of cardiovascular and cerebrovascular disease associated with oral glucocorticoid treatment. This study shows that, in a cohort of patients receiving glucocorticoid by some route, those receiving prescriptions for oral glucocorticoid have a $25 \%$ higher risk of cardiovascular disease than patients with non-oral, non-systemic glucocorticoids after adjusting for confounders. The OR was higher among current users of oral glucocorticoids and patients taking the highest dose. The increased use of oral corticosteroids was greatest for patients with heart failure, was less obvious for patients with ischaemic heart disease, and was not apparent for patients with cerebrovascular disease. Moreover, the increased proportion of current oral glucocorticoid use among patients with cardiovascular disease was observed in patients with different underlying disease indications for glucocorticoid treatment, although it was greater among patients with COPD than among patients with RA or other diseases. Therefore, we found a relatively small but significant increase in risk of cardiovascular events among patients at a high baseline risk. Given the high prevalence of glucocorticoid use in the general population, the number needed to harm-that is, the number of patients needed to be treated with glucocorticoids for one additional outcome event to occuris clinically relevant.

The way cases and controls were identified means that some caution is required in the interpretation of the magnitude of these associations. To ensure active registration of each case in the GPRD and hence to ensure complete outcome data, all participants had to have received at least one prescription during the study period. To minimise bias we chose to compare patients receiving oral glucocorticoids with patients receiving topical glucocorticoids. This means that the estimates in this study reflect a comparison of oral glucocorticoid use with glucocorticoid use by another route, not with non-use of glucocorticoids. Since topical (when frequently used) and inhaled glucocorticoids may also exhibit systemic effects, this approach may slightly underestimate associations with glucocorticoid treatment. Estimation of glucocorticoid exposure is not straightforward, however, since glucocorticoid treatment for chronic disease is typically intermittent over many years. Cumulative dose in intermittent users is difficult to estimate because prescriptions may not always be recorded-for example, if short courses of drugs are issued during hospital admissions-and because the average time between start of follow up and the index date was only about three years. We therefore believe that the best indicator of exposure is the current dose being prescribed to patients who are current users in the three months leading up to their index date; these patients are most likely to be receiving chronic glucocorticoid treatment. Indeed, we found that current users were those patients with the highest number of prescriptions during follow up and who had the highest cumulative dose compared with non-current users of glucocorticoids. In the group of current users we found the strongest associations with cardiovascular disease together with evidence of a dose-response relation.

The ascertainment of cases and controls is also subject to misclassification. All outcome events are related to incident diagnoses. There could have been misclassification with respect to diagnoses of study outcomes but this is likely to be non-differential between cases and controls for cardiovascular and cerebrovascular events. As fluid retention is a known side effect of glucocorticoid use, we cannot exclude the possibility that diagnostic suspicion bias accounts for part of the observed effect for heart failure.

Further, risk factors for cardiovascular disease may be identified and treated more readily among patients being prescribed glucocorticoids. Finally, smoking is more common among patients with COPD and RA and may explain an apparent association between glucocorticoid treatment and cardiovascular disease. To account for these potential confounders, ORs were adjusted for all relevant drug prescriptions and for BMI and smoking. These adjustments had only modest effects on the estimates, suggesting that the 
associations between cardiovascular disease and oral glucocorticoid use are independent of these confounders in this study.

A major methodological concern in observational studies arises from the lack of random allocation of patients to use oral glucocorticoids and hence the potential for confounding by indication. ${ }^{27}$ As both RA and COPD are associated with cardiovascular morbidity and mortality, the underlying disease, rather than exposure to glucocorticoids, may explain the observed association. To address this issue, we examined the consistency of the association between oral glucocorticoid use and cardiovascular disease among patients with different underlying diseases: cases and controls were matched according to the presence of RA, COPD, or the absence of either condition.

The results may also be biased due to confounding by disease severity, as patients using oral glucocorticoids are likely to be more severely affected by the underlying disease. Thus, we adjusted for use of other disease modifying drugs that reflect, at least in part, disease activity in RA. Duration of disease was not a confounder in this study.

Finally, the use of glucocorticoids in RA, COPD, and other conditions may be different. The most frequent indications for oral glucocorticoid treatment besides RA and COPD are skin, neurological, and gastrointestinal tract diseases, ${ }^{1}$ in which patterns of prescription may differ from those in RA and COPD.

Despite these limitations, the results in table 4 show associations between oral glucocorticoid prescription and cardiovascular disease in all groups and show interesting differences between groups. Among patients with COPD, use of oral glucocorticoids was associated more strongly with heart failure than with ischaemic heart disease. Among patients with RA, heart failure and ischaemic heart disease were similarly associated with oral glucocorticoid use. Among patients with neither RA nor COPD, only heart failure was significantly associated with oral glucocorticoid treatment. These data suggest that there is an interaction between the associations of cardiovascular outcome and the underlying disease indication or oral glucocorticoid use, but that both have independent effects.

In all of our analyses, use of oral glucocorticoids was most strongly associated with heart failure. Associations with ischaemic heart disease events were relatively weak and there were no associations with cerebrovascular disease. Sodium retention and increase in extracellular fluid are well known side effects of glucocorticoids and may be responsible for precipitating diagnosis, particularly in patients with cor pulmonale caused by COPD. However, the association between oral glucocorticoid treatment and heart failure was also present among patients with as well as those without COPD who had not received a recorded prescription for glucocorticoids for more than a year before diagnosis, long after any sodium retaining effect would have corrected itself. We can only speculate on the mechanism for this, although it may involve actions of corticosteroids on cardiac remodelling and fibrosis.

Our hypothesis a priori was that oral glucocorticoid use would predict ischaemic heart disease events and strokes because glucocorticoids adversely affect long term risk factors including obesity, blood pressure, plasma lipids, and glucose. However, adjustment for body mass index and antihypertensive, lipid lowering, and hypoglycaemic medications did not substantially attenuate the association between oral glucocorticoid treatment and cardiovascular outcomes. It may be that the risk factors mediating adverse effects of glucocorticoids were not adequately treated in many patients or that subtle changes in risk factors occurred that were insufficient to cause practitioners to initiate treatment but were sufficient to adversely affect outcome. These explanations do not, however, explain the discrepancy between associations of oral glucocorticoid use and occlusive disease in the coronary and cerebrovascular circulation. For patients with stroke and transient ischaemic attack, oral glucocorticoid treatment was associated with a protective effect (table 3 ). This suggests that the mechanisms linking glucocorticoid use and cardiovascular outcome are complex, perhaps reflecting a balance between adverse and potentially protective effects. However, there is also evidence suggesting that patients cured of Cushing's disease still have an increased prevalence of atherosclerosis and maintain cardiovascular risk factors of the active disease, possibly due to residual obesity, insulin resistance syndrome, or both. ${ }^{28} 29$

Our results raise the possibility that general practitioners avoid prescribing oral glucocorticoids to patients with established cardiovascular risk factors. There were no striking differences between users and non-users of oral glucocorticoids, except that patients with diabetes were less likely to have been prescribed oral glucocorticoids. However, diabetes was adjusted for in the multivariate analyses.

\section{Summary}

This large study identified oral glucocorticoid use as a risk factor for heart failure and ischaemic heart disease. Physicians should be aware of the negative vascular effect of glucocorticoids and add this knowledge in balancing positive and negative effects for the individual patient when considering to prescribe glucocorticoids, especially to patients with cardiovascular risk factors. However, this evidence remains observational and only a randomised controlled trial of glucocorticoid treatment versus other disease modifying agents is likely to distinguish the importance of the underlying disease activity from its treatment in predicting cardiovascular outcome.

\section{Authors' affiliations}

P C Souverein, T P Van Staa, A C G Egberts, H G M Leufkens, Department of Pharmacoepidemiology and Pharmacotherapy, Utrecht Institute for Pharmaceutical Sciences, Utrecht, the Netherlands A Berard, Faculty of Pharmacy, University of Montreal, Montreal, Quebec, Canada

T P Van Staa, C Cooper, Medical Research Council, Environmental Epidemiology Unit, Southampton University Hospital, Southampton, UK B R Walker, Department of Medical Sciences, Endocrinology Unit, University of Edinburgh, Edinburgh, UK

\section{REFERENCES}

1 Van Staa TP, Leufkens HG, Abenhaim L, et al. Use of oral corticosteroids in the United Kingdom. QJM 2000;93:105-11.

2 Van Staa TP, Leufkens HG, Abenhaim L, et al. Use of oral corticosteroids and risk of fractures. J Bone Miner Res 2000;15:993-1000.

3 Nashel DJ. Is atherosclerosis a complication of long-term corticosteroid treatment? Am J Med 1986;80:925-9.

4 Maxwell SR, Moots RJ, Kendall MJ. Corticosteroids: do they damage the cardiovascular system? Postgrad Med J 1994;70:863-70.

5 Haynes RC Jr. Adrenocorticotropic hormone; adrenocorticol steroids and their synthetic analogs; inhibitors of the synthesis and actions of adrenocortical hormones. In: Gilman AG, Rall TW, Nies AS, et al, eds. Goodman and Gilman's The pharmacological basis of therapeutics, 8th ed. New York: Pergamon Press, 1990:1431-62.

6 Ross EJ, Linch DC. Cushing's syndrome-killing disease: discriminatory value of signs and symptoms aiding early diagnosis. Lancet 1982;ii:646-9.

7 Watt GC, Harrap SB, Foy CJ, et al. Abnormalities of glucocorticoid metabolism and the renin-angiotensin system: a four-corners approach to the identification of genetic determinants of blood pressure. J Hypertens 1992;10:473-82.

8 Filipovsky J, Ducimetiere $\mathrm{P}$, Eschwege $\mathrm{E}$, et al. The relationship of blood pressure with glucose, insulin, heart rate, free fatty acids and plasma cortisol levels according to degree of obesity in middle-aged men. J Hypertens 1996; 14:229-35.

9 Walker BR, Phillips DI, Noon JP, et al. Increased glucocorticoid activity in men with cardiovascular risk factors. Hypertension 1998;31:891-5. 
10 Rosmond R, Dallman MF, Bjorntorp P. Stress-related cortisol secretion in men relationships with abdominal obesity and endocrine, metabolic and hemodynamic abnormalities. J Clin Endocrinol Metab 1998;83:1853-9.

11 Phillips DI, Barker DJ, Fall CH, et al. Elevated plasma cortisol concentrations: a link between low birth weight and the insulin resistance syndrome? J Clin Endocrinol Metab 1998;83:757-60.

12 Fraser $\mathbf{R}$, Ingram MC, Anderson NH, et al. Cortisol effects on body mass, blood pressure, and cholesterol in the general population. Hypertension 1999:33:1364-8

13 Walker BR, Soderberg S, Lindahl B, et al. Independent effects of obesity and cortisol in predicting cardiovascular risk factors in men and women. $J$ Intern Med 2000;247: 198-204.

14 Reynolds RM, Walker BR, Syddall HE, et al. Altered control of cortisol secretion in adult men with low birth weight and cardiovascular risk factors. $J$ Clin Endocrinol Metab 2001:86:245-50.

15 Ross R. Atherosclerosis: an inflammatory disease. N Engl J Med 1999;340:115-26.

16 Rab ST, King SB 3rd, Roubin GS, et al. oronary aneurysms after stent placement: a suggestion of altered vessel wall healing in the presence of antiinflammatory agents. J Am Coll Cardiol 1991;18:1524-8.

17 Villa AE, Guzman LA, Chen W, et al. Local delivery of dexamethasone for prevention of neointimal proliferation in a rat model of balloon angioplasty. J Clin Invest 1994:93:1243-9.

18 Kirwan JR. The effect of glucocorticoids on joint destruction in rheumatoid arthritis. The Arthritis and Rheumatism Council low-dose glucocorticoid study group. N Engl J Med 1995;333:142-6.

19 Mutru O, Laakso M, Isomaki $\mathrm{H}$, et al. Ten year mortality and causes of death in patients with rheumatoid arthritis. BMJ (Clin Res Ed) 1985;290:1797-9.
20 Raynauld JP. Cardiovascular mortality in rheumatoid arthritis: how harmful are corticosteroids? J Rheumatol 1997;24:415-6.

21 Wallberg-Jonsson S, Johansson H, Ohman ML, et al. Extent of inflammation predicts cardiovascular disease and overall mortality in seropositive rheumatoid arthritis: a retrospective cohort study from disease onset. J Rheumatol 1999;26:2562-71.

22 Goodson N. Coronary artery disease and rheumatoid arthritis. Curr Opin Rheumatol 2002;14:115-20.

23 DeMaria AN. Relative risk of cardiovascular events in patients with rheumatoid arthritis. Am J Cardiol 2002:89:33D-8D.

24 Walley T, Mantgani A. The UK general practice research database. Lancet 1997;350:1097-9.

25 Van Staa TP, Abenhaim L. The quality of information recorded on a UK database of primary care records: a study of hospitalization due to hypoglycemia and other conditions. Pharmacoepidemiol Drug Saf 1994;3:32-4.

26 Greenland S. Modeling and variable selection in epidemiologic analysis. Am J Public Health 1989:79:340-9.

27 Psaty BM, Koepsell TD, Lin D, et al. Assessment and control for confounding by indication in observational studies. J Am Geriatr Soc 1999;47:749-54

28 Colao A, Pivonello R, Spiezia S, et al. Persistence of increased cardiovascular risk in patients with Cushing's disease after five years of successful cure. J Clin Endocrinol Metab 1999:84:2664-72.

29 Faggiano A, Pivonello R, Spiezia S, et al. Cardiovascular risk factors and common carotid artery caliber and stiffness in patients with Cushing's disease during active disease and 1 year after disease remission. J Clin Endocrinol Metab 2003;88:2527-33.

\section{IMAGES IN CARDIOLOGY}

\section{Septic emboli from aortic valve endocarditis}

A 21 year old man, with a history of tissue aortic valve replacement for aortic regurgitation secondary to bicuspid aortic valve, and with good dental hygiene, presented with a four week history of malaise, nausea, anorexia, and fever. In the week preceding hospital admission, he started having abdominal pain.

Physical examination revealed pyrexia, tachycardia, one splinter haemorrhage, soft systolic murmur, a tender left hypochondrium with no rebound or guarding, and good bowel sounds. Initial investigations showed a raised white cell count of $20 \times 10^{9} / \mathrm{l}$, C reactive protein of $300 \mathrm{mg} / \mathrm{dl}$, normal renal function, and three sets of blood cultures which grew Streptococcus viridans. He had a transoesophageal echocardiogram which did not show vegetations but there were features suggestive, but not typical, of aortic root abscess. The patient was treated with benzyl penicillin and gentamicin.

In view of the abdominal pain, he had an abdominal $x$ ray, which showed dilated small and large bowels. A computed tomographic (CT) scan of the abdomen showed splenic infarcts and renal emboli (upper and lower panels), in keeping with septic emboli.

The patient underwent aortic valve replacement the next day. An aortic root abscess was evident. He had an uncomplicated recovery from his surgery and his abdominal complications resolved, without any further intervention. He was discharged seven weeks after admission.

B Skaria

P Kalra

I A Simpson

BinoySkaria@soton.ac.uk

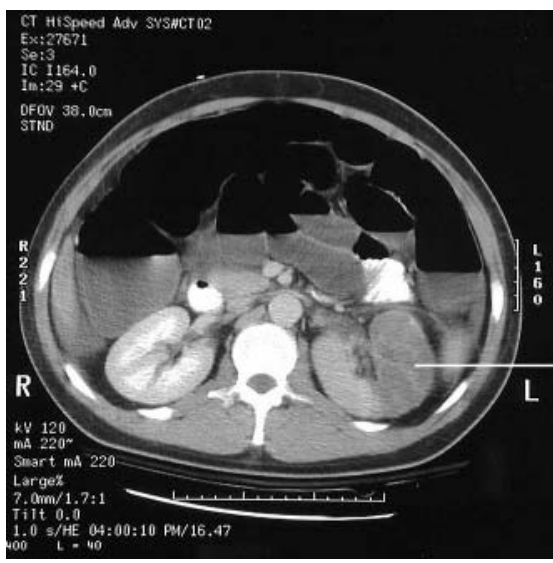

Hypoperfused renal parenchyma and capsular enhancement

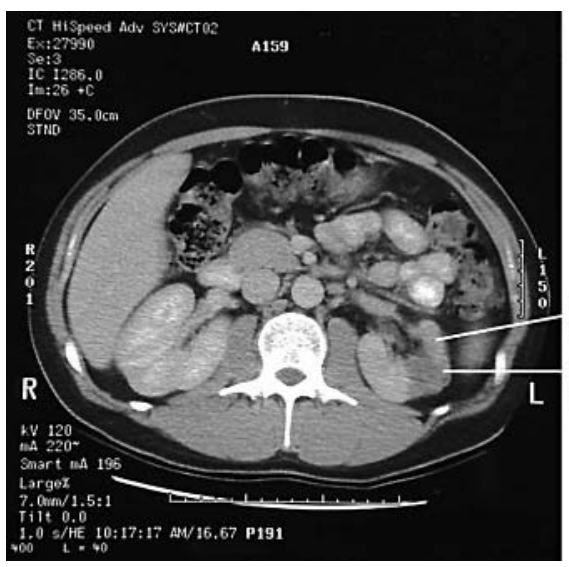

Luxury perfusion from capsular supply Wedge of unperfused renal parenchyma 